\title{
Fuzzy Complex Grassmannian Spaces and their Star Products
}

\author{
Brian P. Dolan ${ }^{1, a}$ and Oliver Jahn ${ }^{2, b}$ \\ ${ }^{a}$ Department of Mathematical Physics \\ NUI Maynooth, Maynooth, Ireland \\ ${ }^{b}$ Dublin Institute for Advanced Studies \\ 10 Burlington Road, Dublin 4, Ireland
}

\begin{abstract}
We derive an explicit expression for an associative star product on noncommutative versions of complex Grassmannian spaces, in particular for the case of complex 2-planes. Our expression is in terms of a finite sum of derivatives. This generalises previous results for complex projective spaces and gives a discrete approximation for the Grassmannians in terms of a non-commutative algebra, represented by matrix multiplication in a finitedimensional matrix algebra. The matrices are restricted to have a dimension which is precisely determined by the harmonic expansion of functions on the commutative Grassmannian, truncated at a finite level. In the limit of infinitedimensional matrices we recover the commutative algebra of functions on the complex Grassmannians.
\end{abstract}

\footnotetext{
${ }^{1}$ bdolan@thphys.may.ie

2jahn@stp.dias.ie
} 
There has recently been much interest in non-commutative geometry, [1, 2, both as a novel direction in string theory [3] and as a new tool in quantum field theory [4]. In the latter approach the theory is formulated on a "fuzzy" space, a series of discrete approximations to a continuous space-time manifold $\mathcal{M}$. The space of fields on each approximation is finite-dimensional, so the fuzzy space serves as a regulator, similar to a lattice. In contrast to the latter, the truncation enjoys all symmetries of the approximated continuous manifold. This has several useful implications including the absence of a doubling problem for chiral fermions [5, 6]. Topologically non-trivial field configurations can be included and the chiral anomaly emerges naturally in this approach [7, 8].

In more detail, a fuzzy space is given by a series of finite-dimensional algebras $A_{L}$ that approximate the commutative algebra of functions on $\mathcal{M}$ in the following way: each $A_{L}$ is identified with a subset of functions on $\mathcal{M}$ and the product in $A_{L}$ induces a (non-commutative) product of functions in this subset, the so-called star product; in the limit $L \rightarrow \infty$, the subset should exhaust the set of all functions and the star product go over to the (commutative) pointwise product of functions. In the present case, the algebras $A_{L}$ are full matrix algebras. The fuzzy spaces are thus matrix geometries, which go over to the usual continuous manifold as the size of the matrix is taken to infinity.

Non-commutative star products are known to exist for every Poisson manifold, in particular for symplectic manifolds [9]. Star products realised by finite-dimensional matrix algebras have been constructed in [10] for all homogeneous Kähler manifolds provided a certain quantisation condition on the metric is satisfied. These algebras can also be obtained using generalised coherent states [1] or the method of orbits for irreducible representations of Lie groups [12, 13]. The relation between these approaches has been discussed in [14] and that to deformation quantisation in [15. [16. For the relation to Fourier transformation on group space see [17]. In terms of functions on the manifold, the above formulations provide an expression of the star product as an integral over the analytical continuation of the functions. This is not very convenient for explicit calculations in non-commutative field theory. An explicit, local formula in terms of a finite number of derivatives is so far only known for complex projective spaces [18] including the case of the fuzzy 2-sphere [19] already treated in [20].

In this paper, we derive an analogous formula for fuzzy complex Grassmannians, that is finite matrix geometry approximations to the usual Grassmannians, $G_{k}^{N} \cong U(N) /[U(k) \times U(N-k)]$, which are homogeneous spaces isomorphic to the space of complex $k$-planes in $\mathbf{C}^{N}$. The matrix geometries consist of matrices acting on the irreducible representation of $S U(N)$ which is given by the $L$-fold Young product of the representation on antisymmetric $k$-tensors. As $L$ is increased the continuous Grassmannian is recovered. The special case $k=1$ requires the $L$-fold symmetric product of the fundamental representation of $S U(N)$, as in [18. The star product for the case $k=2$ is constructed explicitly, using globally well-defined but over-complete co-ordinates on the Grassmannian. The result is expressed as a finite sum over multiple derivatives of the functions, which are decomposed into 
irreducible representations of the stability group $S[U(k) \times U(N-k)]$ acting on the tangent space. It is shown that the star product reduces to the usual commutative product as $L \rightarrow \infty$. The particular case $G_{2}^{4}$ may be of some interest in field theory as it is a symplectic manifold which has $S^{4}$ as a Lagrangian sub-manifold. The corresponding matrix geometries can be viewed as non-commutative versions of $T^{*} S^{4}$, the co-tangent bundle to $S^{4}$ [21]. A star product on the complex Grassmannians as an infinite sum over derivatives is known [22, 23]. However, this formula cannot be restricted to finite-dimensional sub-algebras and therefore cannot serve as a star product on a fuzzy approximation to the manifold.

The layout of the paper is as follows: in section 2 we describe the complex Grassmannians, $G_{k}^{N}$, in terms of projection operators acting in $\mathbf{C}^{N}$, we introduce a set of global co-ordinates which are over-complete and satisfy a set of quadratic constraints which ensures that they indeed describe $G_{k}^{N}$; in section [3] we analyse the algebra of functions in terms of representations of $S U(N)$; in section 4 we describe the finite matrix geometries that define the fuzzy Grassmannians $G_{k, \mathrm{~F}}^{N}$; section $[5$ gives the construction of the star product for the special case $k=2, G_{2, \mathrm{~F}}^{N}$, while section 6 presents a conjecture on the possible form for $k>2$ and some observations on the construction; section 7 gives a summary and conclusions and some technical details are relegated to the appendices.

\section{Complex Grassmannian spaces}

The complex Grassmannian space $G_{k}^{N} \cong U(N) /[U(k) \times U(N-k)]=S U(N) / S[U(k) \times$ $U(N-k)$ ] can be represented as the space of all Hermitean rank- $k$ projectors $\mathcal{P}$ acting on $\mathbf{C}^{N}$. This is easily seen since any such projector can be diagonalised by an element of $U(N)$ and there is still a residual adjoint action of $U(k) \times U(N-k)$ which leaves it invariant. It will be convenient to describe $G_{k}^{N}$ using a redundant set of globally well-defined co-ordinates, $\xi^{A}, A=1, \ldots, N^{2}-1$. First we introduce orthonormal Hermitean generators $t_{A}$ of the Lie algebra of $S U(N)$ satisfying

$$
t_{A} t_{B}=\frac{1}{N} \delta_{A B}+\frac{1}{\sqrt{2}}\left(d_{A B}^{C}+i f_{A B}^{C}\right) t_{C},
$$

where $f_{A B}{ }^{C}$ are the structure constants of $S U(N)$ and $d_{A B}{ }^{C}$ the components of the usual symmetric traceless tensor. The $t_{A}$ are normalised so that $\operatorname{tr}\left(t_{A} t_{B}\right)=\delta_{A B}$. This allows us to parameterise $\mathcal{P}$ in terms of $N^{2}-1$ real parameters $\xi^{A}$,

$$
\mathcal{P}=\frac{k}{N}+\xi^{A} t_{A}
$$

The condition that $\mathcal{P}$ is as projector translates into

$$
\xi^{A} \xi^{A}=\frac{k(N-k)}{N} \quad \text { and } \quad \frac{1}{\sqrt{2}} d_{A B}{ }^{C} \xi^{A} \xi^{B}=\frac{N-2 k}{N} \xi^{C},
$$

and $\xi^{A}$ now parameterise $G_{k}^{N}$ when these conditions are imposed. This construction embeds the Grassmannian in the space of traceless Hermitian matrices, which we identify with $\mathbf{R}^{N^{2}-1}$ parameterised by the unrestricted $\xi^{A}$. 
As discussed in detail in reference [18] for the case of $\mathbf{C P}^{N-1} \equiv G_{1}^{N}$, the complex structure and metric are encoded in a Hermitean projector

$$
K_{B}^{A} \equiv \operatorname{tr}\left[\mathcal{P} t^{A}(1-\mathcal{P}) t_{B}\right]=\frac{1}{2}\left(P_{B}^{A}+i J^{A}{ }_{B}\right)
$$

where $J_{B}^{A}=\sqrt{2} f_{B C}^{A} \xi^{C}$ is the complex structure on $G_{k}^{N}$ and $P^{A}{ }_{B}=P_{B}{ }^{A}=-\left(J^{2}\right)^{A}{ }_{B}$ (indices are raised and lowered with the flat metric $\delta_{B}^{A}$ of $\mathbf{R}^{N^{2}-1}$ ). The matrix $K^{A}{ }_{B}$ projects derivatives with respect to $\xi^{A}$ onto the holomorphic tangent space of the Grassmannian when acting on the right and onto the anti-holomorphic tangent space when acting on the left; so $\nabla_{A} \equiv K_{A}{ }^{B} \partial / \partial \xi^{B}$ is a holomorphic derivative and $\bar{\nabla}_{B} \equiv K^{A}{ }_{B} \partial / \partial \xi^{A}$ an anti-holomorphic one. To see that $K^{A}{ }_{B}$ is a projector we use the completeness relation for the generators,

$$
\left(t_{A}\right)^{i}{ }_{j}\left(t^{A}\right)^{k}{ }_{l}=\delta^{i}{ }_{l} \delta^{k}{ }_{j}-\frac{1}{N} \delta^{i}{ }_{j} \delta^{k}{ }_{l},
$$

to show that

$$
K^{A}{ }_{B} t^{B}=\mathcal{P} t^{A}(1-\mathcal{P}) \quad \text { and } \quad t_{B} K^{B}{ }_{A}=(1-\mathcal{P}) t_{A} \mathcal{P} .
$$

(These relations will prove very useful in the ensuing analysis.) Examining the real and imaginary parts of $K_{B}^{A}$ separately reveals that $P=-J^{2}$ and $P J=J P=J$. This means that $P$ itself is a projector onto the tangent space of $G_{k}^{N}$, with rank $2 k(N-k)$. An important observation for the following analysis is that the differential operators $K_{A}^{B} \partial / \partial \xi^{B}$ commute with the constraints (3), as they must do, since $K$ projects onto the tangent space. This can also be proven using (6). It is this fact that allows us to use the global co-ordinates, rather than local co-ordinates, in the final differential expression for the star product.

Covariant derivatives can be constructed by projecting derivatives with respect to the flat coordinates $\xi^{A}$ to the tangent space. Multiple covariant holomorphic derivatives are thus defined as

$$
\nabla_{A_{1}} \cdots \nabla_{A_{n}} f(\xi) \equiv\left(K_{A_{1}}^{B_{1}} \cdots K_{A_{n}}^{B_{n}}\right)\left(\partial_{B_{1}} K_{B_{2}}{ }^{C_{2}} \partial_{C_{2}} \cdots K_{B_{n}}{ }^{C_{n}} \partial_{C_{n}} f(\xi)\right)
$$

where $\partial_{A}=\partial / \partial \xi^{A}$. In our case there is a simplification because

$$
K^{A B} K^{C D}\left(\partial_{B} K_{D}^{E}\right)=0
$$

which follows from the definition (44) of $K$ and the completeness relation (5). It implies

$$
\nabla_{A_{1}} \cdots \nabla_{A_{n}} f(\xi)=\left(K_{A_{1}}^{B_{1}} \cdots K_{A_{n}}^{B_{n}}\right)\left(\partial_{B_{1}} \cdots \partial_{B_{n}} f(\xi)\right)
$$

\section{Harmonic analysis}

In order to obtain a finite-dimensional truncation of the space of functions on the Grassmannian, which is compatible with the symmetries, we decompose the space of functions into irreducible representations of the isometry group $G=S U(N)$.

We think of $G_{k}^{N}$ as the space $G / H$ of (right) cosets in $G$ with respect to $H=$ $S[U(k) \times U(N-k)]$, the subset of matrices in $U(k) \times U(N-k)$ with unit determinant. 
Functions on $G / H$ can thus be considered as functions on $G$ that are invariant under the left action of $H$. They transform under $G$ according to the right action.

The full space of functions on $G$ is spanned by the matrix elements $D_{M M^{\prime}}^{J}$ of all irreducible unitary representations $J$ of $G$. Decomposing into irreducible representations of $H$, we may write the first component index as $M=(n, j, m)$ where $j$ labels the irreducible representations of $H, m$ the corresponding components and $n$ distinguishes copies of equivalent representations. The left action of $H$ is then given by

$$
D_{(n, j, m) M^{\prime}}^{J}\left(h^{-1} g\right)=\sum_{m^{\prime \prime}} D_{(n, j, m)\left(n, j, m^{\prime \prime}\right)}^{J}\left(h^{-1}\right) D_{\left(n, j, m^{\prime \prime}\right) M^{\prime}}^{J}(g) .
$$

The $H$-invariant matrix elements are those for which the first index corresponds to the trivial representation of $H$, labelled for instance by $j=m=0$. The space of functions on $G / H$ is thus spanned by the matrix elements $D_{(n, 0,0)}^{J} M^{\prime}$.

Under the right action of $G$,

$$
D_{(n, 0,0) M^{\prime}}^{J}\left(g g^{\prime}\right)=\sum_{M^{\prime \prime}} D_{(n, 0,0) M^{\prime \prime}}^{J}(g) D_{M^{\prime \prime} M^{\prime}}^{J}\left(g^{\prime}\right),
$$

so, for fixed $J$ and $n$, the $D_{(n, 0,0) M^{\prime}}^{J}$ span the vector space of the representation $J$ of $G$. The space of functions on $G_{k}^{N}$ thus contains all irreducible representations of $S U(N)$ that contain the trivial representation upon restriction to $S[U(k) \times U(N-k)]$. The multiplicities are given by the multiplicity of the trivial representation in the restriction.

We will describe representations of $S U(N)$ by Young diagrams. These will be denoted by their symbols $J=\left[j_{1}, j_{2}, \ldots, j_{N-1}\right]$ where $j_{i}$ denotes the number of columns of height $i$ of the diagram. ${ }^{3}$ The fundamental representation, for instance, has $J=[1,0, \ldots]$ and the adjoint one $J=[1,0, \ldots, 0,1]$. Note that the complex conjugate representation is given by $J^{*}=\left[j_{N-1}, \ldots, j_{1}\right]$. In appendix [ we show that a representation $J$ of $S U(N)$ contains the trivial representation of $S[U(k) \times$ $U(N-k)]$ if and only if it appears in the direct product

$$
M_{L} \equiv\left[\mathbf{0}_{k-1}, L, \mathbf{0}_{N-k-1}\right] \otimes\left[\mathbf{0}_{N-k-1}, L, \mathbf{0}_{k-1}\right]
$$

for $L \geq n / N$ where $n=\sum_{i} j_{i}$ is the number of boxes in the diagram $J$ and $\mathbf{0}_{k-1}$ stands for $k-1$ zero entries. The multiplicity in the restriction is the same as that in the direct product. The $M_{L}$ satisfy $M_{1} \subset M_{2} \subset \cdots$, so they provide a hierarchy of truncations of the space of functions on the Grassmannian.

The representation $\left[\mathbf{0}_{k-1}, L, \mathbf{0}_{N-k-1}\right]$ is the Young product of $L$ anti-symmetric $k$-tensors, for instance $\bigoplus$ for $k=2, L=5$. As an example, for $N=6$ and $k=2$, the first truncation is

$$
\begin{gathered}
M_{1}=[0,1,0,0,0] \otimes[0,0,0,1,0]=[0,0,0,0,0] \oplus[1,0,0,0,1] \oplus[0,1,0,1,0] \\
\theta \otimes
\end{gathered}
$$

\footnotetext{
${ }^{3}$ Note that this symbol is different from the highest weight vector sometimes used to describe a diagram, the entries of the latter being the number of boxes in each row.
} 
the second is

$$
\begin{aligned}
& M_{2}=[0,2,0,0,0] \otimes[0,0,0,2,0]=[0,0,0,0,0] \oplus[1,0,0,0,1] \oplus[0,1,0,1,0] \\
& \oplus[2,0,0,0,2] \oplus[1,1,0,1,1] \oplus[0,2,0,2,0] .
\end{aligned}
$$

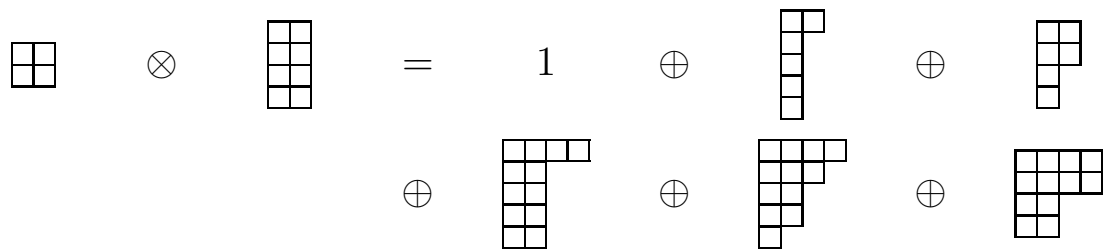

Although not needed in the following, we would like to state for illustrational purposes that the full harmonic analysis on $G_{k}^{N}$ (the "union" of all $M_{L}$ ) is given by the representations

$$
J= \begin{cases}{\left[m_{1}, \ldots, m_{k}, 0, \ldots, 0, m_{k}, \ldots, m_{1}\right]} & \text { if } 2 k<N, \\ {\left[m_{1}, \ldots, m_{k-1}, 2 m_{k}, m_{k-1}, \ldots, m_{1}\right]} & \text { if } 2 k=N,\end{cases}
$$

with $m_{i}=0,1, \ldots$, each representation occurring once. The case $2 k>N$ can be obtained by replacing $k$ by $N-k$, since $G_{k}^{N} \cong G_{N-k}^{N}$. The representations (15) correspond to Young diagrams which can be obtained by putting a diagram with at most $k$ rows next to its conjugate (which has at least $N-k \geq k$ boxes in any column), as can be verified for the examples given above. This result is also derived in appendix $\mathrm{C}$

\section{Matrix geometry}

In the previous section, we have obtained a series of truncations of the space of functions on the Grassmannian. We will now see that these carry a natural product. The representation content $M_{L}$ of a given truncation can in fact be realised as an algebra of matrices in a representation $J$ of $S U(N)$ : since such matrices transform under $S U(N)$ by conjugation, they form the representation space of $J \otimes J^{*}$; so $M_{L}$, as introduced in eq. (12), is equivalent to the space of matrices in the representation $\left[\mathbf{0}_{k-1}, L, \mathbf{0}_{N-k-1}\right]$. Since the matrix product respects the action of $S U(N)$, the algebra $M_{L}$ has the same symmetries as $G_{k}^{N}$.

In order to obtain the corresponding product of (truncated) functions, we shall now construct an injective map from $M_{L}$ to the space of functions on the Grassmannian which also respects the group action (an equivariant map). This map automatically provides the notions of differentiation and integration needed for the construction of actions: one just has to map the corresponding notions for functions back to matrices. Equivariance guarantees that they are compatible with the truncation. The map will also provide a non-commutative product for functions in the image of $M_{L}$, the star product. If the star product tends to the point-wise product in the limit $L \rightarrow \infty$, we have succeeded in constructing a fuzzy $G_{k}^{N}$. Since we will restrict ourselves to $k=2$ in the following sections, we present the map only for this case. The generalisation to other values of $k$ should be obvious. 
For $G_{2}^{N}$ the basic building block will be the anti-symmetric representation corresponding to $L=1$. The first non-trivial truncation of functions therefore requires using $[N(N-1) / 2] \times[N(N-1) / 2]$ matrices. A function on $G_{2}^{N}$ is associated with such a matrix $\hat{F}$ by restricting the tensor product of the fundamental projector (2) to the anti-symmetric representation $\theta$,

$$
\rho \equiv(\mathcal{P} \otimes \mathcal{P})_{a}
$$

and constructing

$$
F_{1}(\xi)=\operatorname{tr}[\rho(\xi) \hat{F}]
$$

Since $\mathcal{P}$ has rank $2, \rho$ has rank 1: let the plane onto which $\mathcal{P}$ projects be spanned by the vectors $\vec{v}$ and $\vec{w} ; \rho$ then projects onto the 1-dimensional subspace of the representation space of $\boxminus$ spanned by the anti-symmetric product of $\vec{v}$ and $\vec{w}$ (an explicit proof is given in appendix $(\mathrm{B})$. A general truncation requires taking the $L$-fold (Young) product $[0, L, 0, \ldots]=\bigoplus_{\cdots} \ldots$ of $\boxminus$, which has dimension $n_{L}^{N}=\frac{(N+L-1) !(N+L-2) !}{(N-1) ! L !(N-2) !(L+1) !}$, and using $n_{L}^{N} \times n_{L}^{N}$ matrices. Equations (13) and (14), for instance, show the decomposition of $15 \times 15$ and $105 \times 105$ matrices as harmonics for $G_{2}^{6}$. In the following we shall drop trailing zeros in symbols of representations, so the above representations will be denoted by $[0, L]$. The Young product can be obtained as a component of the symmetric tensor product, so a projector can be constructed by restricting the $L$-fold tensor product of $\rho$ to the representation $[0, L]$,

$$
\rho_{L}=(\overbrace{\rho \otimes \cdots \otimes \rho}^{L \text { times }})_{[0, L]},
$$

(of course $\rho_{1}=\rho$ ) and a function can be associated with any $n_{L}^{N} \times n_{L}^{N}$ matrix $\hat{F}$ by

$$
F_{L}(\xi)=\operatorname{tr}\left[\rho_{L}(\xi) \hat{F}\right]
$$

Appendix B contains a proof that the map (19) is injective.

The matrix geometries introduced here coincide with those obtained from complex line bundles [10] or generalised coherent states [11], see [14, 15]. $F_{L}$ is usually called the covariant symbol of the operator $\hat{F}$ in these formulations, and injectivity is well known and follows from an analyticity argument. The relation with coherent states will be discussed in some more detail in appendix B.

\section{$5 \quad$ Star product on $G_{2}^{N}$}

Multiplication of truncated functions on the Grassmannian can now be defined using matrix multiplication. The star product of two functions, $F_{L}=\operatorname{tr}\left(\rho_{L} \hat{F}\right)$ and $G_{L}=$ $\operatorname{tr}\left(\rho_{L} \hat{G}\right)$, is obtained from the matrix product through the map

$$
\left(F_{L} \star G_{L}\right)(\xi)=\operatorname{tr}\left[\rho_{L}(\xi) \hat{F} \hat{G}\right]
$$

By construction this is an associative product and it keeps within the class of functions truncated at level $L$. Our aim is to find an explicit expression for this star 
product, purely in terms of $F_{L}$ and $G_{L}$ and their derivatives, thus eliminating the explicit reference to matrices.

By orthonormality of the matrix elements in the representation $[0, L]$, $\int \mathrm{d} \mu(g) D_{M_{1} M_{2}}^{[0, L]}\left(g^{-1}\right) D_{M_{3} M_{4}}^{[0, L]}(g)=\left(1 / n_{L}^{N}\right) \delta_{M_{1} M_{4}} \delta_{M_{2} M_{3}}, \hat{F}$ can be expanded as

$$
\hat{F}=\int \mathrm{d} \mu(g) \tilde{F}(g) D^{[0, L]}(g)
$$

with

$$
\tilde{F}(g) \equiv n_{L}^{N} \operatorname{tr}\left[D^{[0, L]}\left(g^{-1}\right) \hat{F}\right] .
$$

Inserting this into (19), we obtain

$$
F_{L}(\xi)=\int \mathrm{d} \mu(g) \omega_{L}(\xi, g) \tilde{F}(g)
$$

with

$$
\omega_{L}(\xi, g) \equiv \operatorname{tr}\left[\rho_{L}(\xi) D^{[0, L]}(g)\right]
$$

and the star product can be expressed as

$$
\left(F_{L} \star G_{L}\right)(\xi)=\int \mathrm{d} \mu(g) \int \mathrm{d} \mu\left(g^{\prime}\right) \omega_{L}\left(\xi, g g^{\prime}\right) \tilde{F}(g) \tilde{G}\left(g^{\prime}\right) .
$$

We seek an expression for the star product in terms of derivatives acting on $F_{L}(\xi)$ and $G_{L}(\xi)$. By eqs. (25) and (23), this can be achieved by deriving an expression for $\omega_{L}\left(\xi, g g^{\prime}\right)$ in terms of derivatives of $\omega_{L}(\xi, g)$ and $\omega_{L}\left(\xi, g^{\prime}\right)$ with respect to $\xi$. The latter is greatly facilitated by the observation that $\omega_{L}$ can be expressed in terms of $\omega_{1}$,

$$
\omega_{L}(\xi, g)=\left[\omega_{1}(\xi, g)\right]^{L},
$$

because, by eq. (18), $\rho_{L}$ factorises into rank-1 projectors $\rho$ and $D^{[0, L]}$ acts as a direct product as well. The reason behind this relation is that the representation $[0, L]$ when projected to $S[U(2) \times U(N-2)]$ by $\rho_{L}$ factorises as $[0,1]^{L}$ since all other irreducible components of the product involve tensors that are anti-symmetric in 3 or more indices and therefore vanish in $S U(2)$.

As a first step, we have to find an expression for $\omega_{1}\left(\xi, g g^{\prime}\right)$. This is a straightforward but somewhat lengthy exercise. It is deferred to appendix $\mathrm{A}$ and yields

$$
\omega_{1}\left(\xi, g g^{\prime}\right)=\omega_{1}(\xi, g)\left(1+\overleftarrow{\partial}_{A} K^{A B} \vec{\partial}_{B}+\frac{1}{4} \overleftarrow{\partial}_{A} \overleftarrow{\partial}_{B} K^{A C} K^{B D} \vec{\partial}_{C} \vec{\partial}_{D}\right) \omega_{1}\left(\xi, g^{\prime}\right)
$$

where $\partial_{A}=\partial / \partial \xi^{A}$ and $K$ is the projector onto the holomorphic tangent space introduced in eq. (44). Substituting (27) in (25) and interchanging differentiation with respect to $\xi$ with integration over $g$ and $g^{\prime}$, we now have the star product at level one,

$$
\left(F_{1} \star G_{1}\right)(\xi)=F_{1}(\xi)\left(1+\overleftarrow{\partial}_{A} K^{A B} \vec{\partial}_{B}+\frac{1}{4} \overleftarrow{\partial}_{A} \overleftarrow{\partial}_{B} K^{A C} K^{B D} \vec{\partial}_{C} \vec{\partial}_{D}\right) G_{1}(\xi)
$$


For higher $L$ we have to consider $\omega_{L}=\left(\omega_{1}\right)^{L}$. Equation (27) implies

$$
\begin{array}{r}
\omega_{L}\left(\xi, g g^{\prime}\right)=\sum_{n+m \leq L} \frac{L !}{n ! m !(L-n-m) !}\left(\omega \omega^{\prime}\right)^{L-n-m}\left[\left(\partial_{A} \omega\right) K^{A B}\left(\partial_{B} \omega^{\prime}\right)\right]^{n} \\
\times\left[\frac{1}{4}\left(\partial_{C} \partial_{D} \omega\right) K^{C E} K^{D F}\left(\partial_{E} \partial_{F} \omega^{\prime}\right)\right]^{m}
\end{array}
$$

where we have used the abbreviations $\omega \equiv \omega_{1}(\xi, g)$ and $\omega^{\prime} \equiv \omega_{1}\left(\xi, g^{\prime}\right)$. The righthand side of this equation has to be expressed in terms of multiple derivatives acting on $\omega_{L}(\xi, g)$ and $\omega_{L}\left(\xi, g^{\prime}\right)$. It contains several different terms with a given number of derivatives. This means that we have to distinguish components of multiple derivatives of $\omega_{L}$.

To this end, we decompose multiple holomorphic derivatives $\nabla_{A_{1}} \cdots \nabla_{A_{n}} \omega_{L}$ as defined in eq. (91) with respect to irreducible representations of the stability group $H$ which acts on the tangent space. It will be sufficient to consider the subgroup $H_{0}=S U(2) \times S U(N-2)$ of $H$. Representations of $H_{0}$ will be denoted by $\left(J, J^{\prime}\right)$ where $J$ is a representation of the first factor and $J^{\prime}$ one of the second. To find the representation content of a single holomorphic derivative $\nabla_{A}=K_{A}{ }^{B} \partial_{B}$, note that the fundamental representation [1] of $S U(N)$ decomposes as

$$
\left.[1]\right|_{H_{0}}=([1],[0]) \oplus([0],[1])
$$

into the fundamental representations of $S U(2)$ and $S U(N-2)$ upon restriction to $H_{0}$. The two components can be obtained by projection with $\mathcal{P}$ and $1-\mathcal{P}$. Now use eqs. (4I) and (5) to write $\nabla_{A}$ in terms of (anti-)fundamental indices,

$$
\left(t^{A}\right)^{i}{ }_{j} \nabla_{A} f(\xi)=\left[(1-\mathcal{P}) t^{B} \mathcal{P}\right]_{j}^{i} \partial_{B} f(\xi) .
$$

The matrix $t^{B} \partial_{B} f$ transforms like the traceless component of $[1] \times[1]^{*}$ under $S U(N)$. Since the index $i$ is projected by $1-\mathcal{P}$ to $([0],[1])$ while $j$ is projected by $\mathcal{P}$ to $([1],[0])^{*}$, we find that the holomorphic derivative transforms like $([0],[1]) \otimes$ $([1],[0])^{*}=\left([1]^{*},[1]\right)$. Note that tracelessness is guaranteed by the projections in eq. (31). By the same reasoning, an anti-holomorphic derivative $\bar{\nabla}_{A}=K^{B}{ }_{A} \partial_{B}$ transforms like $\left([1],[1]^{*}\right)$. A multiple holomorphic derivative transforms like the symmetric tensor product of $n$ copies of the representation $\left([1]^{*},[1]\right)$. In order to obtain an explicit expression for the decomposition of this product, it turns out to be useful to first decompose the tensor product of $n$ copies of the fundamental representation of $S U(N)$.

This can be done by considering the action of the symmetric group $S_{n}$, whose elements permute the factors in the tensor product [28]. The latter can then be decomposed into irreducible representations of $S U(N) \times S_{n}$ with the help of character projection operators. They provide the following decomposition of unity,

$$
1=\sum_{|J|=n} P_{J} \quad \text { where } \quad P_{J} \equiv \frac{d_{J}}{n !} \sum_{\pi \in S_{n}} \chi_{J}(\pi) \pi
$$

Here, the sum is over all Young diagrams with $n$ boxes, $\chi_{J}$ is the character of the symmetric group in the representation $J$ and $d_{J}$ the dimension of that representation. 
We will only need

$$
d_{[l, m]}=\frac{(l+2 m) !(l+1)}{(l+m+1) ! m !} .
$$

The projectors $P_{J}$ are orthogonal, $P_{J} P_{J^{\prime}}=\delta_{J, J^{\prime}} P_{J}$. They are discussed in more detail in appendix $\mathrm{D}$. When acting on tensors, $P_{J}$ projects onto a direct product of the representations of $S U(N)$ and the symmetric group given by $J$. Consequently the image of $P_{J}$ contains $d_{J}$ copies of the $S U(N)$ representation $J$.

This decomposition can be used to write the projector onto symmetric tensors with adjoint indices as

$$
\frac{1}{n !} \delta_{A_{1}}^{\left\{B_{1}\right.} \cdots \delta_{A_{n}}^{\left.B_{n}\right\}}=\frac{1}{n !} \sum_{|J|=n} \operatorname{tr}\left[\left(t_{A_{1}} \otimes \cdots \otimes t_{A_{n}}\right)\left(t^{\left\{B_{1}\right.} \otimes \cdots \otimes t^{\left.B_{n}\right\}}\right) P_{J}\right]
$$

where curly brackets denote symmetrisation. The projector from general adjoint tensors to symmetric holomorphic tensors (or anti-holomorphic tensors when acting to the left) can now be decomposed as

$$
\frac{1}{n !} K_{A_{1}}{ }^{\left\{B_{1}\right.} \cdots K_{A_{n}}^{\left.B_{n}\right\}}=\sum_{l+2 m=n} K_{[l, m] A_{1} \ldots A_{n}}{ }^{B_{1} \ldots B_{n}}
$$

with projection operators

$$
\begin{aligned}
K_{J}^{A_{1} \ldots A_{n}, B_{1} \ldots B_{n}} & =\frac{1}{n !} \operatorname{tr}\left[\left(t^{A_{1}} \otimes \cdots \otimes t^{A_{n}}\right)\left(t^{\left\{C_{1}\right.} \otimes \cdots \otimes t^{\left.C_{n}\right\}}\right) P_{J}\right] K_{C_{1}}{ }^{B_{1}} \cdots K_{C_{n}}{ }^{B_{n}} \\
& =\frac{1}{n !} \operatorname{tr}\left[\left(t^{A_{1}} \otimes \cdots \otimes t^{A_{n}}\right) P_{J}(1-\mathcal{P})^{\otimes n}\left(t^{\left\{B_{1}\right.} \otimes \cdots \otimes t^{\left.B_{n}\right\}}\right) \mathcal{P}^{\otimes n} P_{J}\right] .
\end{aligned}
$$

The second expression has been obtained by inserting eq. (6) for the contraction of $K_{C}{ }^{B}$ with $t^{C}$ and generating a second projector using the fact that $P_{J}=P_{J}^{2}$ commutes with symmetric tensors. To see that $K_{J}$ is a projector, one has to use the completeness relation (5) for the generators $t^{A}$ and the fact that $P_{J}$ and $K_{A}{ }^{B}$ are projectors. The first expression shows that, when acting to the right, $K_{J}$ projects onto the holomorphic tangent space. The second expression reveals that the holomorphic tensors thus obtained are projected to a sum of equivalent irreducible representations of $H_{0}=S U(2) \times S U(N-2)$ : the first indices of the $n$ matrices $t^{B_{i}}$ are projected to $[1]^{\otimes n}$ of $S U(N-2)$ and then symmetrised according to the Young diagram $J$, the second indices are projected to $[1]^{* \otimes n}$ of $S U(2)$ and symmetrised in the same way; the result is a sum of representations equivalent to $\left(J^{*}, J\right)$ of $H_{0}$. Since $P_{J}$ projects onto $d_{J}$ copies of $J$ corresponding to different symmetrisations and the symmetrisation on both sides of the symmetric $t^{\left\{B_{1}\right.} \otimes \cdots \otimes t^{\left.B_{n}\right\}}$ has to be the same, $K_{J}$ projects onto $d_{J}$ copies of $\left(J^{*}, J\right)$. When acting to the left, $K_{J}$ projects onto the anti-holomorphic tangent space since the factors $K$ commute with the trace. The result is projected to $d_{J}$ copies of the representation $\left(J, J^{*}\right)$.

These findings imply that $K_{\left[j_{1}, j_{2}, \ldots\right]}$ vanishes if $j_{i} \neq 0$ for any $i>2$ as can be seen explicitly by noting that the second $P_{J}$ is multiplied by $\mathcal{P}{ }^{\otimes j}$ in eq. (36) and $\mathcal{P}$ is a rank-2 projector. This fact has been used to restrict the sum in eq. (35). Note 
that $K_{[1]}^{A, B}=K^{A B}$ and

$$
\begin{aligned}
K_{[0,1]}^{A B, C D}=\frac{1}{4}\{ & \operatorname{tr}\left[\mathcal{P} t^{A}(1-\mathcal{P}) t^{C}\right] \operatorname{tr}\left[\mathcal{P} t^{B}(1-\mathcal{P}) t^{D}\right] \\
& \left.-\operatorname{tr}\left[\mathcal{P} t^{A}(1-\mathcal{P}) t^{C} \mathcal{P} t^{B}(1-\mathcal{P}) t^{D}\right]\right\}+(C \leftrightarrow D) .
\end{aligned}
$$

A useful observation is that $K_{[l, m]}$ factorises when contracted with arbitrary symmetric tensors $S$ and $T$,

$$
\begin{aligned}
& S_{\left\{A_{1} \ldots A_{n}\right\}} K_{[l, m]}^{A_{1} \ldots A_{n}, B_{1} \ldots B_{n}} T_{\left\{B_{1} \ldots B_{n}\right\}} \\
= & d_{[l, m]} S_{\left\{A_{1} \ldots A_{n}\right\}} K_{[l]}^{A_{1} \ldots A_{l}, B_{1} \ldots B_{l}} K_{[0,1]}^{A_{l+1} A_{l+2}, B_{l+1} B_{l+2}} \cdots K_{[0,1]}^{A_{n-1} A_{n}, B_{n-1} B_{n}} T_{\left\{B_{1} \ldots B_{n}\right\}},
\end{aligned}
$$

or, in index-free notation, $S K_{[l, m]} T=d_{[l, m]} S\left(K_{[l]} \otimes K_{[0,1]}^{\otimes m}\right) T$ where $d_{[l, m]}$ is given in eq. (33). This equation is proved in appendix D.

The irreducible components of the derivatives of $\omega_{L}=\omega^{L}$ are calculated in appendix $\mathrm{E}$ in index-free notation, the result is

$$
\begin{aligned}
& \left(\partial^{l+2 m} \omega^{L}\right) K_{[l, m]}\left(\partial^{l+2 m} \omega^{L L}\right) \\
& \quad=c_{l, m}^{(L)}\left(\omega \omega^{\prime}\right)^{L-l-m}\left[(\partial \omega)^{l} K_{[l]}\left(\partial \omega^{\prime}\right)^{l}\right]\left[\frac{1}{4}(\partial \partial \omega) K_{[0,1]}\left(\partial \partial \omega^{\prime}\right)\right]^{m}
\end{aligned}
$$

where

$$
c_{l, m}^{(L)}=\left(\frac{L !(L+1) !}{(L-l-m) !(L+1-m) !}\right)^{2} d_{[l, m]} .
$$

The crucial ingredient in the derivation is the following relation between single and double derivatives of $\omega$,

$$
K^{A C} K^{B D} \omega \partial_{C} \partial_{D} \omega=K_{[0,1]}^{A B, C D} \omega \partial_{C} \partial_{D} \omega=2 K_{[0,1]}^{A B, C D} \partial_{C} \omega \partial_{D} \omega,
$$

which can be obtained by use of eqs. (50) and (60) in a somewhat tedious calculation. In addition, since $\omega$ is only quadratic in $\xi^{A}$, one has $\partial_{A} \partial_{B} \partial_{C} \omega=0$.

With the tools provided, it is easy to bring the terms in the expression (29) for $\omega_{L}(\xi, g g)$ into the form (39). Owing to eq. (41), we have

$$
\frac{1}{4} \partial_{C} \partial_{D} \omega K^{C E} K^{D F} \partial_{E} \partial_{F} \omega^{\prime}=\partial_{C} \partial_{D} \omega K_{[0,1]}^{C E, D F} \partial_{E} \partial_{F} \omega^{\prime} .
$$

Now we use the decomposition (35) together with the factorisation property (38) to write

$$
\left(\partial_{A} \omega K^{A B} \partial_{B} \omega^{\prime}\right)^{n}=\sum_{l+2 i=n} d_{[l, i]}\left[(\partial \omega)^{l} K_{[l]}\left(\partial \omega^{\prime}\right)^{l}\right]\left[(\partial \partial \omega) K_{[0,1]}\left(\partial \partial \omega^{\prime}\right)\right]^{i}\left(\omega \omega^{\prime}\right)^{i} .
$$

Inserting this into eq. (29) and combining equivalent terms, we obtain

$$
\left.\omega_{L}\left(\xi, g g^{\prime}\right)=\sum_{l+m \leq L} \tilde{c}_{l, m}^{(L)}\left(\omega \omega^{\prime}\right)^{L-l-m}\left[(\partial \omega)^{l} K_{[l]}\left(\partial \omega^{\prime}\right)^{l}\right)\right]\left[(\partial \partial \omega) K_{[0,1]}\left(\partial \partial \omega^{\prime}\right)\right]^{m}
$$


with

$$
\tilde{c}_{l, m}^{(L)}=\sum_{i=0}^{\min (m, L-l-m)} \frac{L !}{(l+2 i) !(m-i) !(L-l-m-i) !} d_{[l, i]} .
$$

The sum can be performed using the identity

$$
\sum_{i=0}^{\min (p, n)}\left(\begin{array}{c}
p \\
i
\end{array}\right)\left(\begin{array}{c}
q \\
n-i
\end{array}\right)=\left(\begin{array}{c}
p+q \\
n
\end{array}\right),
$$

one finds

$$
\tilde{c}_{l, m}^{(L)}=\frac{L !(L+1) !}{(L-l-m) !(L+1-m) !(l+2 m) !} d_{[l, m]} .
$$

Now the terms have exactly the form (39) and we can write

$$
\omega_{L}\left(\xi, g g^{\prime}\right)=\sum_{l+m \leq L} a_{l, m}^{(L)}\left(\partial^{l+2 m} \omega_{L}(\xi, g)\right) K_{[l, m]}(\xi)\left(\partial^{l+2 m} \omega_{L}\left(\xi, g^{\prime}\right)\right)
$$

with

$$
a_{l, m}^{(L)}=\frac{(L-l-m) !(L+1-m) !}{L !(L+1) !(l+2 m) !} .
$$

Inserting eq. (48) into eq. (25) and interchanging differentiation with respect to $\xi$ with integration over $g$ and $g^{\prime}$, we obtain the final expression for the star product,

$$
\begin{aligned}
& \left(F_{L} \star G_{L}\right)(\xi)= \\
& \sum_{l+m \leq L} a_{l, m}^{(L)}\left(\partial_{A_{1}} \cdots \partial_{A_{l+2 m}} F_{L}(\xi)\right) K_{[l, m]}^{A_{1} \ldots A_{l+2 m}, B_{1} \ldots B_{l+2 m}}(\xi)\left(\partial_{B_{1}} \cdots \partial_{B_{l+2 m}} G_{L}(\xi)\right) .
\end{aligned}
$$

Recall that $K_{[l, m]}$ projects the derivatives on its right to holomorphic ones in the irreducible representation $[l, m]^{*} \otimes[l, m]$ of the subgroup $S U(2) \times S U(N-2)$ of the stability group and those on its left to anti-holomorphic ones in the complex conjugate representation.

Since the star product (50) is ultimately derived from a matrix product it is guaranteed to be associative, by construction. For large $L, a_{l, m}^{(L)} \sim L^{-l-2 m}$, so all terms except $l=m=0$ are suppressed and the star product tends to the point-wise product. We conclude that the series of matrix geometries introduced in section 4 indeed constitutes a fuzzy version of the complex Grassmannians $G_{2}^{N}$. As a consistency check note that restricting the sum to $m=0$ reduces this to the known result for $\mathbf{C P}^{N-1}$, [18].

\section{Observations on the construction and generali- sation to $k>2$}

The above results suggest a natural generalisation to the case $k>2$, although we have not verified it: the definition of matrix geometries in section 4 generalises immediately to Young products of antisymmetric $k$-tensors; in eq. (27), we expect 
analogous terms with up to $k$ derivatives acting on each side, and eq. (150) is expected to contain projections $K_{\left[j_{1} \ldots j_{k}\right]}$ with $\sum_{i} j_{i} \leq L$; a natural extension of the combinatorial factor (49) would be

$$
a_{J}^{(L)}=\frac{1}{|J| !} \prod_{i} \frac{1}{L+r_{i}-c_{i}}
$$

where the product runs over all boxes of the Young diagram $J$ and $r_{i}$ and $c_{i}$ are the row and column of box $i$.

Returning to the particular case $k=2$, it might be more convenient, for practical purposes, to express $K_{[l, m]}$ in terms of $K$ and $K_{[0,1]}$ alone by using the inverse of the decomposition (35): we can add factors of $K_{[0,1]}$ on both sides of (35) and use (38) to write

$$
S\left(K^{\otimes(p-2 n)} \otimes K_{[0,1]}^{\otimes n}\right) T=\sum_{m=n}^{[p / 2]} M_{n, m}^{(p)} S\left(K_{[p-2 m]} \otimes K_{[0,1]}^{\otimes m}\right) T
$$

where $S$ and $T$ are symmetric tensors and $M_{n, m}^{(p)}=d_{[p-2 m, m-n]}$. For fixed $p, M^{(p)}$ can be considered as a triangular matrix with $M_{n, m}^{(p)}=0$ if $n>m$. It can be inverted by use of the relation

$$
\sum_{m=n}^{n^{\prime}} d_{[p-2 m, m-n]} e_{p-2 n^{\prime}, n^{\prime}-m}=\delta_{n, n^{\prime}} \quad \text { with } \quad e_{i, j}=(-1)^{j}\left(\begin{array}{c}
i+j \\
j
\end{array}\right)
$$

which can be derived by standard techniques. We find $\left(M^{(p)}\right)_{m, n}^{-1}=e_{p-2 n, n-m}$ for $m \leq n$ and 0 otherwise. The inverse of eq. (52) therefore is

$$
S K_{[p-2 m, m]} T=d_{[p-2 m, m]} \sum_{n=m}^{[p / 2]} e_{p-2 n, n-m} S\left(K^{\otimes(p-2 n)} \otimes K_{[0,1]}^{\otimes n}\right) T
$$

where the factorisation property (38) has been used on the left-hand side. Inserting this into eq. (50) and re-expanding indices, we obtain an alternative formula for the star product,

$$
\begin{aligned}
& \left(F_{L} \star G_{L}\right)(\xi)=\sum_{n+m \leq L} b_{n, m}^{(L)}\left(\partial_{A_{1}} \cdots \partial_{A_{n+2 m}} F_{L}(\xi)\right)\left(K^{A_{1} B_{1}}(\xi) \cdots K^{A_{n} B_{n}}(\xi)\right. \\
& \left.\quad \times K_{[0,1]}^{A_{n+1} A_{n+2}, B_{n+1} B_{n+2}}(\xi) \cdots K_{[0,1]}^{A_{n+2 m-1} A_{n+2 m}, B_{n+2 m-1} B_{n+2 m}}(\xi)\right)\left(\partial_{B_{1}} \cdots \partial_{B_{n+2 m}} G_{L}(\xi)\right)
\end{aligned}
$$

where (33), (49) and (53) have been combined into

$$
b_{n, m}^{(L)}=\sum_{i=0}^{\min (m, L-n-m)}(-1)^{i} \frac{(L-n-m-i) !(L+1+i-m) !(n+i) !(n+2 i+1)}{L !(L+1) !(n+m+i+1) !(m-i) ! i ! n !} .
$$

$K$ and $K_{[0,1]}$ are explicitly given by

$$
\begin{aligned}
K^{A B} & =\frac{1}{2}\left(-J^{A C} J_{C}{ }^{B}+i J^{A B}\right) \quad \text { with } \quad J^{A B}=\sqrt{2} f^{A B}{ }_{C} \xi^{C}, \\
K_{[0,1]}^{A B, C D} & =\left(\frac{N-2}{4 N}\left(\delta_{E}^{A} \delta_{F}^{B}+\delta_{F}^{A} \delta_{E}^{B}\right)-\frac{1}{4}\left(d_{E}^{A G} d_{G F}^{B}+d_{F}^{A G} d_{G E}^{B}\right)\right) K^{E C} K^{F D} .
\end{aligned}
$$




\section{Conclusions}

We have extended an explicit, local description of fuzzy matrix geometries beyond the known examples of $\mathbf{C P}_{\mathrm{F}}^{N-1}$ to complex Grassmannians $G_{k}^{N} \cong U(N) /[U(k) \times$ $U(N-k)]$. The geometry of the Grassmannian is conveniently described in terms of $N^{2}-1$ globally defined co-ordinates $\xi^{A}$, satisfying the quadratic constraints (3), and a rank- $k$ projector $\mathcal{P}$ defined in (2). The fuzzy Grassmannians $G_{k, \mathrm{~F}}^{N}$ are defined by algebras of $n_{L}^{N} \times n_{L}^{N}$ matrices, $\hat{F}$, where $n_{L}^{N}$ is the dimension of the irreducible representation of $S U(N)$ which is the $L$-fold Young product of the $k$-fold antisymmetric representation and has Young diagram

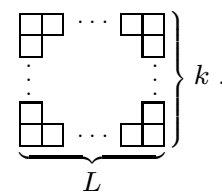

Such matrices are mapped injectively to functions using a rank-1 projector constructed from the $k$-fold anti-symmetric tensor product of $\mathcal{P}, \rho=(\mathcal{P} \otimes \cdots \otimes \mathcal{P})_{a}$ as the $L$-fold product $\rho_{L}=(\rho \otimes \cdots \otimes \rho)_{\left[\mathbf{0}_{k-1}, L, \mathbf{o}_{N-k-1}\right]}$. The map is given by

$$
F_{L}(\xi)=\operatorname{tr}\left[\rho_{L}(\xi) \hat{F}\right]
$$

The set of functions thus obtained constitute a truncation of the harmonic expansion of a general function on $G_{k}^{N}$.

An associative star product between two such functions can then be defined using matrix multiplication as

$$
\left(F_{L} \star G_{L}\right)(\xi)=\operatorname{tr}\left[\rho_{L}(\xi) \hat{F} \hat{G}\right]
$$

The right-hand side can be re-expressed as a (bi-)differential operator on $F_{L}(\xi)$ and $G_{L}(\xi)$ and is given explicitly, for $k=2$, in eq. (50),

$$
\begin{aligned}
& \left(F_{L} \star G_{L}\right)(\xi)= \\
& \quad \sum_{l+m \leq L} a_{l, m}^{(L)}\left(\partial_{A_{1}} \cdots \partial_{A_{l+2 m}} F_{L}(\xi)\right) K_{[l, m]}^{A_{1} \ldots A_{l+2 m}, B_{1} \ldots B_{l+2 m}}(\xi)\left(\partial_{B_{1}} \cdots \partial_{B_{l+2 m}} G_{L}(\xi)\right)
\end{aligned}
$$

where $a_{l, m}^{(L)}$ is given by eq. (49)) and $K_{[l, m]}$ by eq. (36). $K_{[l, m]}$ projects the multiple derivatives on $F_{L}$ and $G_{L}$ to irreducible representations of the stability group $H=$ $S[U(2) \times U(N-2)]$. It includes a projection of the derivatives to the tangent space of $G_{2}^{N}$ in the embedding space parameterised by $\xi$. Tangent vectors transform under $H$ and the multiple tangential derivative forms a direct product representation which is reducible. The derivatives on $G_{L}$ are projected by $K_{[l, m]}$ to the component equivalent to the irreducible representation $[l, m]^{*} \otimes[l, m]$ of $S U(2) \times S U(N-2) \subset H$. Here, $[l, m]$ stands for a Young product of $m$ copies of the anti-symmetric representation $\exists$ and $l$ copies of the fundamental representation $\square$, for instance $[4,3]=\square \square$. The derivatives on $F_{L}$ are projected to the complex conjugate representation. The projection implies that all derivatives on $G_{L}$ are holomorphic while those on $F_{L}$ are 
anti-holomorphic. They can in fact be replaced by covariant derivatives in local co-ordinates [18. When the sum is restricted to $m=0$ this star product reduces to the $k=1$ case of $\mathbf{C P}^{N-1}$, [18].

An even more explicit expression for the star product, possibly better suited for practical purposes, is given in eq. (55).

Explicit, local formulas for the star product prove very useful in perturbative calculations in non-commutative field theory [30] and might also provide new insights in string theory where fuzzy versions of co-adjoint orbits appear as world volume geometries of D-branes in WZW models [31, 32].

\section{Acknowledgements}

The authors wish to thank A. P. Balachandran, Denjoe O'Connor and P. Prešnajder for useful discussions and the Department of Physics, CINVESTAV, Mexico for their hospitality during a fruitful visit. The work of OJ has been supported by the Alexander von Humboldt Foundation through a Feodor Lynen Research Fellowship (grant number V-3-FLF-1068701).

\section{A Kernel at level 1}

We derive an expression for the kernel $\omega_{1}\left(\xi, g g^{\prime}\right)$ in terms of derivatives of $\omega_{1}(\xi, g)$ and $\omega_{1}\left(\xi, g^{\prime}\right)$ with respect to $\xi$. Inserting eq. (16) and the definition of the antisymmetric representation, $D^{[0,1]}(g)=(g \otimes g)_{a}$, into eq. (24) and abbreviating $\omega_{1}$ as $\omega$, we obtain

$$
\omega(\xi, g)=\operatorname{tr}\left[(\mathcal{P} \otimes \mathcal{P})_{a}(g \otimes g)_{a}\right] \quad \text { with } \quad \mathcal{P}=\frac{2}{N}+\xi^{A} t_{A}
$$

The trace of a tensor product in the anti-symmetric representation can be expressed as

$$
\operatorname{tr}\left[(A \otimes B)_{a}\right]=\frac{1}{2}[\operatorname{tr}(A) \operatorname{tr}(B)-\operatorname{tr}(A B)] .
$$

A crucial identity, $\mathcal{P}_{[l}^{i} \mathcal{P}_{m}^{j} \mathcal{P}^{k}{ }_{n]}=0$, follows from the fact that $\mathcal{P}$ is a rank-2 projector. It implies

$$
\begin{aligned}
\operatorname{tr}(\mathcal{P} A) \operatorname{tr}(\mathcal{P} B) \operatorname{tr}(\mathcal{P} C)-\operatorname{tr}(\mathcal{P} A \mathcal{P} B) \operatorname{tr}(\mathcal{P} C)-\operatorname{tr}(\mathcal{P} A \mathcal{P} C) \operatorname{tr}(\mathcal{P} B) \\
-\operatorname{tr}(\mathcal{P} B \mathcal{P} C) \operatorname{tr}(\mathcal{P} A)+\operatorname{tr}(\mathcal{P} A \mathcal{P} B \mathcal{P} C)+\operatorname{tr}(\mathcal{P} B \mathcal{P} A \mathcal{P} C)=0 .
\end{aligned}
$$

Since

$$
\partial_{A} \omega=\operatorname{tr}\left[\left(t_{A} \otimes \mathcal{P}\right)_{a}(g \otimes g)_{a}\right]+\operatorname{tr}\left[\left(\mathcal{P} \otimes t_{A}\right)_{a}(g \otimes g)_{a}\right]
$$

we can use (6) to show

$$
\partial_{A} \omega(\xi, g) K^{A B} \partial_{B} \omega\left(\xi, g^{\prime}\right)=2 \operatorname{tr}\left[(\mathcal{P} \otimes \mathcal{P})_{a}(g \otimes g)_{a}(\mathcal{P} \otimes(1-\mathcal{P}))_{a}\left(g^{\prime} \otimes g^{\prime}\right)_{a}\right] .
$$

Similarly, for the second derivative

$$
\begin{aligned}
\partial_{A} \partial_{B} \omega(\xi, g) K^{A C} K^{B D} \partial_{C} \partial_{D} \omega\left(\xi, g^{\prime}\right) \\
=4 \operatorname{tr}\left[(\mathcal{P} \otimes \mathcal{P})_{a}(g \otimes g)_{a}((1-\mathcal{P}) \otimes(1-\mathcal{P}))_{a}\left(g^{\prime} \otimes g^{\prime}\right)_{a}\right] .
\end{aligned}
$$


Since $\rho_{1}=(\mathcal{P} \otimes \mathcal{P})_{a}$ is a rank-1 projector, the simple product can be written as

$$
\omega(\xi, g) \omega\left(\xi, g^{\prime}\right)=\operatorname{tr}\left[(\mathcal{P} \otimes \mathcal{P})_{a}(g \otimes g)_{a}(\mathcal{P} \otimes \mathcal{P})_{a}\left(g^{\prime} \otimes g^{\prime}\right)_{a}\right] .
$$

Using $1=(\mathcal{P} \otimes \mathcal{P})_{a}+(\mathcal{P} \otimes(1-\mathcal{P}))_{a}+((1-\mathcal{P}) \otimes \mathcal{P})_{a}+((1-\mathcal{P}) \otimes(1-\mathcal{P}))_{a}$, eqs. (62), (63) and (64) can be combined to

$$
\omega\left(\xi, g g^{\prime}\right)=\omega(\xi, g)\left(1+\overleftarrow{\partial}_{A} K^{A B} \vec{\partial}_{B}+\frac{1}{4} \overleftarrow{\partial}_{A} \overleftarrow{\partial}_{B} K^{A C} K^{B D} \vec{\partial}_{C} \vec{\partial}_{D}\right) \omega\left(\xi, g^{\prime}\right)
$$

which is the desired expression.

\section{B Coherent states}

We show that the projector $\rho_{L}$ as given in eq. (18) has rank 1, and we present a simple argument for why the map from matrices to functions is injective.

To this end, we require a more explicit representation for $\rho_{L}$. The vector space of the irreducible representation of $S U(N)$ with symbol $J=[0, L]$ can be realised as a sub-space with certain symmetry properties of the space of $2 L$-index tensors. We construct it as the image of a Young symmetriser. We first assign tensor indices to the boxes in the Young diagram of the representation by putting the numbers $1,2, \ldots, 2 L$ in ascending order into one column after the other, for instance $\frac{1135517}{24468}$ for $[0,4]$. The Young symmetriser is now defined as

$$
\begin{gathered}
Y_{[0, L]}=\frac{2^{L}}{L+1} \mathcal{A}_{L} \mathcal{S}_{L}, \\
\mathcal{A}_{L}=\prod_{i=1}^{L}\left[\frac{1}{2}\left(1-\tau_{i}\right)\right], \quad \mathcal{S}_{L}=\frac{1}{L !} \sum_{\pi_{1} \in R_{1}} \pi_{1} \frac{1}{L !} \sum_{\pi_{2} \in R_{2}} \pi_{2}
\end{gathered}
$$

where $\tau_{i}$ interchanges the two boxes of the $i^{\text {th }}$ column of the diagram and $R_{i}$ denotes the set of permutations that permute the boxes of row $i$. So $\mathcal{S}_{L}$ symmetrises the rows of the diagrams, while $\mathcal{A}_{L}$ anti-symmetrises the columns. Both are symmetric projectors. The Young symmetriser is a projector, $Y_{[0, L]}^{2}=Y_{[0, L]}$, but not symmetric.

Operators in the vector space of $[0, L]$ can be unambiguously described as operators $\hat{F}$ on $2 L$-tensors that satisfy

$$
\hat{F} Y_{[0, L]}=Y_{[0, L]} \hat{F}=\hat{F} .
$$

Now we can prove that $\rho_{L}$ has rank 1 by expressing the rank-2 projector $\mathcal{P}$ in terms of an orthonormal basis $|\varphi\rangle,|\psi\rangle$ of the complex plane onto which it projects,

$$
\mathcal{P}=|\varphi\rangle\langle\varphi|+| \psi\rangle\langle\psi| .
$$

The level-1 projector $\rho$ was defined in (16) as the projection of the tensor product of $\mathcal{P}$ with itself to the anti-symmetric representation $[0,1]$. Since $Y_{[0,1]}$ reduces to a single anti-symmetrisation,

$$
\rho \equiv(\mathcal{P} \otimes \mathcal{P})_{a}=(\mathcal{P} \otimes \mathcal{P}) Y_{[0,1]}=|\varphi \psi\rangle\langle\varphi \psi|
$$


where

$$
|\varphi \psi\rangle \equiv \frac{1}{\sqrt{2}}(|\varphi\rangle|\psi\rangle-|\psi\rangle|\varphi\rangle) .
$$

For the projector at level $L$, we obtain

$$
\rho_{L}=(\rho \otimes \cdots \otimes \rho) Y_{[0, L]}=|\varphi \psi\rangle^{L}\left\langle\left.\varphi \psi\right|^{L} Y_{[0, L]}\right.
$$

The state $|\varphi \psi\rangle$ completely characterises the plane that corresponds to a point in $G_{2}^{N}$. It is therefore natural that it occurs as a fundamental object in the construction. The states $|\varphi \psi\rangle^{L}$ coincide, up to a conventional phase, with the generalised coherent states discussed in [11]. Since $\hat{F} Y_{[0, L]}=Y_{[0, L]} \hat{F}$,

$$
F_{L}(\xi)=\left\langle\left.\varphi \psi\right|^{L} \hat{F} \mid \varphi \psi\right\rangle^{L} .
$$

So $F_{L}$ is the covariant symbol, as defined in [1], of the operator $\hat{F}$.

This expression can be used to show that the map is injective. We have to show that $\hat{F}$ can be reconstructed from $F_{L}$. Since $|\varphi \psi\rangle^{L}=2^{L / 2} \mathcal{A}_{L}(|\varphi\rangle|\psi\rangle)^{L}$,

$$
F_{L}(\xi)=2^{L}\left(\left\langle\varphi|\langle\psi|)^{L} \mathcal{A}_{L} \hat{F} \mathcal{A}_{L}(|\varphi\rangle|\psi\rangle)^{L} .\right.\right.
$$

Due to the anti-symmetrisation between $|\varphi\rangle$ and $|\psi\rangle$ this function can be homogeneously extended to general (non-orthonormal) $|\varphi\rangle$ and $|\psi\rangle$. We choose $|\varphi\rangle=$ $\sum_{n=1}^{N} a_{n}|n\rangle$ and $|\psi\rangle=\sum_{n=1}^{N} b_{n}|n\rangle$ with canonical basis vectors $|n\rangle$. By differentiating with respect to $a, b$ and their complex conjugates, all matrix elements of $\mathcal{S}_{L} \mathcal{A}_{L} \hat{F} \mathcal{A}_{L} \mathcal{S}_{L}$ can be obtained. Using the symmetry (67), we obtain $\mathcal{S}_{L} \hat{F}$ and thus also $\frac{2^{L}}{L+1} \mathcal{A}_{L} \mathcal{S}_{L} \hat{F}=\hat{F}$.

\section{Restrictions and direct products}

We shall derive the relation between the restriction of representations of $G=S U(N)$ to $H=S[U(k) \times U(N-k)]$ and the direct product of certain representations used in section 3. In this appendix, we will allow for columns of height $N, J=$ $\left[j_{1}, j_{2}, \ldots, j_{N}\right]$, in diagrams describing representations of $S U(N)$. These do not lead to new representations, since representations differing only by $j_{N}$ are unitarily equivalent, but this generalisation will make formulas much simpler. We embed $H$ into $S U(N)$ as

$$
\left(\begin{array}{cc}
\mathrm{e}^{\mathrm{i}(N-k) \varphi} U^{\prime} & 0 \\
0 & \mathrm{e}^{-\mathrm{i} k \varphi} U^{\prime \prime}
\end{array}\right)
$$

where $U^{\prime} \in S U(k)$ and $U^{\prime \prime} \in S U(N-k)$ and $\mathrm{e}^{\mathrm{i} \varphi} \in U(1)$. This shows that $H=$ $[S U(k) \times S U(N-k) \times U(1)] / Z_{n}$ where $n$ is the least common multiple of $k$ and $N-k$. Representations of $H$ can thus be considered as representations of $S U(k) \times$ $S U(N-k) \times U(1)$ that represent $Z_{n}$ trivially. This fixes the charge $q$ of the $U(1)$ factor $\mathrm{e}^{\mathrm{i} q \varphi}$ of the representation modulo $n$. We will denote these representations as $\left(J^{\prime}, J^{\prime \prime}\right)_{q}$ where $J^{\prime}$ and $J^{\prime \prime}$ are symbols of $S U(k)$ and $S U(N-k)$ representations, respectively, and $q$ is the charge of the $U(1)$ representation. 
The restriction of an $S U(N)$ representation to $H$ can be written as

$$
\left.J\right|_{H}=\bigoplus_{J^{\prime}, J^{\prime \prime}} m_{J^{\prime}, J^{\prime \prime}}^{J}\left(J^{\prime}, J^{\prime \prime}\right)_{(N-k)\left|J^{\prime}\right|-k\left|J^{\prime \prime}\right|} .
$$

Here, $|J|=\sum_{i} j_{i}$ is the number of boxes in the diagram $J$ and we assume that the diagrams have been chosen such that the total number of boxes in $J^{\prime}$ and $J^{\prime \prime}$ is the same as in $J$,

$$
|J|=\left|J^{\prime}\right|+\left|J^{\prime \prime}\right|
$$

Note that the $U(1)$ representation is determined by the $S U(k) \times S U(N-k)$ representation, so the multiplicities $m_{J^{\prime}, J^{\prime \prime}}^{J}$ are the same as for the restriction from $S U(N)$ to the latter. It is known $(24,25]$, also see [26, 27]) that these can be obtained from the decomposition of the direct product of the $S U(N)$ representations with diagrams $J^{\prime}$ and $J^{\prime \prime}$,

$$
J^{\prime} \otimes J^{\prime \prime}=\bigoplus_{J} m_{J^{\prime}, J^{\prime \prime}}^{J} J \quad \text { in } S U(N) .
$$

Here, again, the restriction (74) on the number of boxes applies. Note that in eq. (75) $J^{\prime}$ and $J^{\prime \prime}$ are interpreted as $S U(N)$ representations while they are interpreted as $S U(k)$ respectively $S U(N-k)$ representations in eq. (73)).

We are interested in the case where the trivial representation of $H$ appears on the right-hand side of (73). This means that $J^{\prime}$ and $J^{\prime \prime}$ only have columns of height $k$ and $N-k$, respectively, $J^{\prime}=\left[\mathbf{0}_{k-1}, L^{\prime}, \mathbf{0}_{N-k-1}\right]$ and $J^{\prime \prime}=\left[\mathbf{0}_{N-k-1}, L^{\prime \prime}, \mathbf{0}_{k-1}\right]$ where $\mathbf{0}_{k-1}$ stands for $k-1$ zero entries, etc. In addition, the $U(1)$ charge has to vanish, $(N-k)\left|J^{\prime}\right|=k\left|J^{\prime \prime}\right|$. Since $\left|J^{\prime}\right|=L^{\prime} k$ and $\left|J^{\prime \prime}\right|=L^{\prime \prime}(N-k)$, this implies $L^{\prime}=L^{\prime \prime} \equiv L$, so that $J^{\prime}$ and $J^{\prime \prime}$ are complex conjugate representations of $S U(N)$. We conclude that a representation of $S U(N)$ contains the trivial representation of $S[U(k) \times U(N-k)]$ if and only if it appears in the decomposition of the direct product

$$
M_{L} \equiv\left[\mathbf{0}_{k-1}, L, \mathbf{0}_{N-k-1}\right] \otimes\left[\mathbf{0}_{N-k-1}, L, \mathbf{0}_{k-1}\right]
$$

for some $L$. The multiplicities are given by the multiplicities $m_{\left[\mathbf{0}_{k-1}, L, \mathbf{0}_{N-k-1}\right],\left[\mathbf{0}_{N-k-1}, L, \mathbf{0}_{k-1}\right]}^{J}$ in the product. Note that the multiplicity does not depend on the number $j_{N}$ of columns of height $N$ in the diagram $J$ chosen for a given representation. This means that a representation appears in $M_{L}$ if and only if $N L \geq|J|$ where $J$ is the minimal diagram $\left(j_{N}=0\right)$ of the representation. Therefore $M_{L} \subset M_{L^{\prime}}$ if $L<L^{\prime}$.

\section{Decomposition of the direct product}

For illustrational purposes, we will explicitly perform the decomposition of the direct product (76) into irreducible representations. We can assume $k \leq N-k$ since $G_{k}^{N} \cong G_{N-k}^{N}$. The decomposition is achieved by Young diagram techniques. Recall the rules for decomposing the direct product of two irreducible representations of $S U(N)$ [28]:

1. Label each box in the second diagram by its row number. 


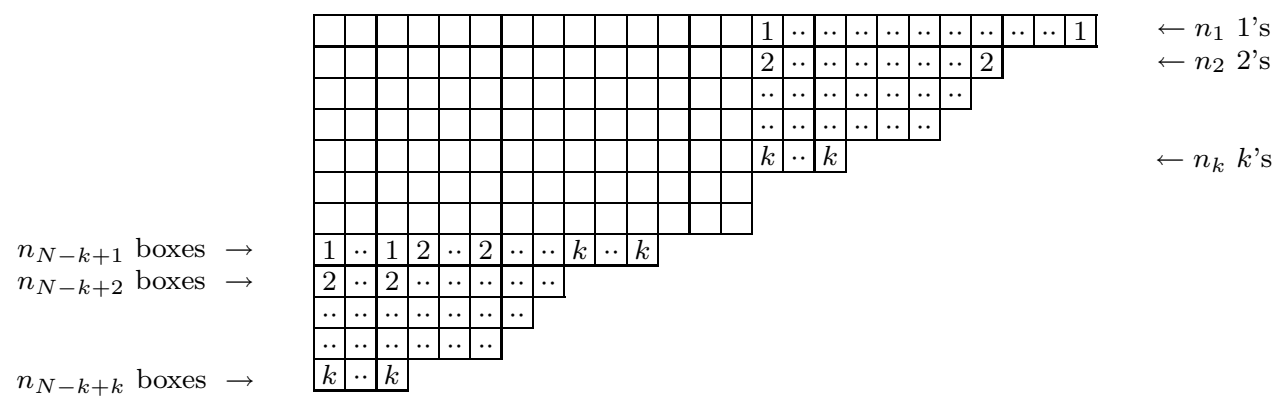

Figure 1: Decomposition of the tensor product $\left[\mathbf{0}_{N-k-1}, L, \mathbf{0}_{k-1}\right] \otimes\left[\mathbf{0}_{k-1}, L, \mathbf{0}_{N-k-1}\right]$.

2. Attach all boxes with 1's to the first diagram, then all boxes with 2's and so on, such that

(a) at all stages the intermediate diagram corresponds to an irreducible representation of $S U(N)$, i.e. all columns start in the first row and are connected and the height of the columns monotonically decreases from left to right,

(b) no column contains any number more than once, and

(c) when counted from the right, the $n$-th $i$ does not appear before the $n$-th $i-1$.

Applying these rules to our case, we have to attach boxes with $L$ copies of each of the numbers $1, \ldots, k$ to a rectangle of height $N-k$ and width $L$. This is indicated in figure 1. We have already anticipated some facts about the resulting distribution of boxes, which we will explain now. The number of 1's in the first row has been denoted by $n_{1}$. The remaining $L-n_{1} 1$ 's have to be in the $N-k+1$-st row. Denoting the number of 2's in the second row by $n_{2}$, there can be at most $n_{1}-n_{2}$ 2 's in row $N-k+1$, because of rule $2 \mathrm{c}$. There must thus be at least $L-n_{1} 2$ 's in row $N-k+2$. However, owing to rule $2 \mathrm{~b}$, the number of 2's in that row is at most $L-n_{1}$. Therefore, there have to be exactly $n_{1}-n_{2} 2$ 's in row $N-k+1$ and $L-n_{1}$ 2 's in row $N-k+2$. By the same reasoning, we find that the number of $i$ 's in row $N-k+l$ for $l>0$ is equal to the number of $i-l+1$ 's in the row $N-k+1$ which is in turn equal to $n_{i-l}-n_{i-l+1}$ if $i \geq l$ and 0 if $i<l$ (we have put $n_{0} \equiv L$ ). Adding up, we find for the number of boxes in row $N-k+l$,

$$
n_{N-k+l}=\sum_{i=l}^{k}\left(n_{i-l}-n_{i-l+1}\right)=L-n_{k-l+1} \quad \text { for } \quad l=1, \ldots, k .
$$

Graphically, this means that the subdiagram of columns $L+1, L+2, \ldots$ combines, after a rotation by $\pi$, with the remainder of the diagram to a rectangle of width $L$ and height $N$. In terms of symbols $J=\left[j_{1}, \ldots, j_{N}\right]$, where $j_{i}$ is the number of columns of height $i$, this implies

$$
J= \begin{cases}{\left[m_{1}, \ldots, m_{k}, 0, \ldots, 0, m_{k}, \ldots, m_{1}\right]} & \text { if } 2 k<N \\ {\left[m_{1}, \ldots, m_{k-1}, 2 m_{k}, m_{k-1}, \ldots, m_{1}\right]} & \text { if } 2 k=N\end{cases}
$$


with $m_{i}=n_{i}-n_{i+1}$ where $n_{k+1} \equiv 0$. All non-negative values of $m_{i}$ satisfying $\sum_{i=1}^{k} m_{i}=n_{1} \leq L$ occur. Furthermore, each diagram can be obtained in only one way, since it is determined by the numbers $n_{i}(i=1, \ldots, k)$. So all multiplicities equal 1.

\section{Symmetric group}

In this appendix, we shall provide a proof of the factorisation property (38). On the way, we will recall some facts about representations of the symmetric group and the associated projectors $P_{J}$ used in the text. These projectors can be considered as elements of the group algebra $\mathbf{R}\left[S_{j}\right] \equiv \operatorname{span} S_{n}=\left\{A=\sum_{\pi \in S_{n}} A_{\pi} \pi \mid A_{\pi} \in \mathbf{R}\right\}$, the set of formal linear combinations of group elements. The vector space $\mathbf{R}\left[S_{j}\right]$ carries a representation of the algebra $\mathbf{R}\left[S_{j}\right]$ whose action is given by left multiplication. This representation restricts to a representation of the subgroup $S_{n}$ of $\mathbf{R}\left[S_{j}\right]$. It is usually called the regular representation. Sometimes it is convenient to identify $\mathbf{R}\left[S_{j}\right]$ with the algebra $F\left(S_{n}\right)$ of functions on the group $S_{n}$ by setting $A(\pi) \equiv A_{\pi}$. The action of a group element $\pi$ is then given by $(\pi A)(\sigma)=A\left(\pi^{-1} \sigma\right) . F\left(S_{n}\right)$ can in fact be considered as the dual vector space of $\mathbf{R}\left[S_{j}\right]$ since each function on the group can be linearly and uniquely extended to a function on $\mathbf{R}\left[S_{j}\right]$. The identification of $\mathbf{R}\left[S_{j}\right]$ with its dual space can be obtained from the inner product

$$
\langle A, B\rangle \equiv \sum_{\pi \in S_{n}} A_{\pi} B_{\pi}=\frac{1}{n !} \operatorname{tr}\left(A^{\mathrm{T}} B\right)
$$

by putting $A(B)=\langle A, B\rangle$. The trace in eq. (179) is over the regular representation and we have set $A^{\mathrm{T}}=\sum_{\pi} A_{\pi} \pi^{-1}$.

Of particular importance are the central elements of $\mathbf{R}\left[S_{j}\right]$, that are invariant under conjugation with any group element $\pi \in S_{n}, A=\pi A \pi^{-1}$. They correspond to class functions, i.e. functions that depend only on the conjugacy class of their argument. An orthogonal basis in the subspace of class functions is given by the characters $\chi_{J}$ associated with the irreducible representations $J$ of $S_{n}$,

$$
\left\langle\chi_{J}, \chi_{J^{\prime}}\right\rangle=n ! \delta_{J J^{\prime}}
$$

So every class function can be expanded as

$$
A=\sum_{J} A_{J} \chi_{J} \quad \text { with } \quad A_{J}=\frac{1}{n !}\left\langle\chi_{J}, A\right\rangle .
$$

To each $A \in \mathbf{R}\left[S_{j}\right]$, one can associate a central element $\bar{A}$ by averaging with respect to conjugation,

$$
\bar{A} \equiv \frac{1}{n !} \sum_{\pi \in S_{n}} \pi A \pi^{-1}=\frac{1}{n !} \sum_{J}\left\langle\chi_{J}, A\right\rangle \chi_{J}
$$

where we have used (81) and the invariance of $\chi_{J}$ under conjugation.

The regular representation is in general reducible. It contains each irreducible representation $J$ with a multiplicity that is given by the dimension $d_{J}$ of the representation. The component containing all copies of an irreducible representation $J$ 
can be obtained as the image of the symmetric projection operator introduced in eq. (32), in the dual picture,

$$
P_{J}=\frac{d_{J}}{n !} \chi_{J}
$$

The decomposition of unity

$$
1=\sum_{J} P_{J}
$$

provides a decomposition of $\mathbf{R}\left[S_{j}\right]$ into orthogonal subspaces [29].

Now we will show how the averaged tensor product of two projectors $P_{J_{1}}$ and $P_{J_{2}}$ onto irreducible representations of $S_{n_{1}}$ and $S_{n_{2}}$ can be expressed in terms of irreducible projectors. $P_{J_{1}} \otimes P_{J_{2}}$ can be extended to $\mathbf{R}\left[S_{j}\right]$ where $n=n_{1}+n_{2}$. By (82), we have

$$
\overline{P_{J_{1}} \otimes P_{J_{2}}}=\sum_{J} a_{J} \chi_{J}
$$

with

$$
a_{J}=\frac{1}{n !}\left\langle\chi_{J}, P_{J_{1}} \otimes P_{J_{2}}\right\rangle
$$

The restriction of $\chi_{J}$ to $(\pi, \sigma) \in S_{n_{1}} \times S_{n_{2}}$ decomposes into irreducible characters as

$$
\chi_{J}(\pi, \sigma)=\sum_{J_{1}, J_{2}} c_{J_{1} J_{2}}^{J} \chi_{J_{1}}(\pi) \chi_{J_{2}}(\sigma)
$$

where $c_{J_{1} J_{2}}^{J} \in \mathbf{Z}$ are multiplicities or Clebsch-Gordan coefficients. With (80) we get

$$
a_{J}=\frac{1}{n !} \sum_{J_{1}^{\prime}, J_{2}^{\prime}} c_{J_{1}^{\prime} J_{2}^{\prime}}^{J}\left\langle\chi_{J_{1}^{\prime}}, P_{J_{1}}\right\rangle\left\langle\chi_{J_{2}^{\prime}}, P_{J_{2}}\right\rangle=\frac{d_{J_{1}} d_{J_{2}}}{n !} c_{J_{1} J_{2}}^{J}
$$

and therefore

$$
\overline{P_{J_{1}} \otimes P_{J_{2}}}=\sum_{J} \frac{d_{J_{1}} d_{J_{2}}}{n !} c_{J_{1} J_{2}}^{J} \chi_{J}=\sum_{J} \frac{d_{J_{1}} d_{J_{2}}}{d_{J}} c_{J_{1} J_{2}}^{J} \overline{P_{J}} .
$$

By iteration, this result can be generalised to multiple products,

$$
\frac{1}{d_{J_{1}} \cdots d_{J_{m}}} \overline{P_{J_{1}} \otimes \cdots \otimes P_{J_{m}}}=\sum_{J} c_{J_{1} \ldots J_{m}}^{J} \frac{1}{d_{J}} \overline{P_{J}} .
$$

Note that symmetrisation with respect to $S_{n}$ implies symmetrisation with respect to $S_{n^{\prime}} \subset S_{n}$

$$
\overline{A \otimes B \otimes C}=\overline{A \otimes \overline{B \otimes C}} .
$$

Now we can prove eq. (38). The right-hand side of this equation can be written as a single trace like in eq. (36) but with $P_{J}$ replaced by $P_{[l]} \otimes P_{[0,1]}^{\otimes m}$. Owing to the symmetric tensors $S$ and $T$ all factors in the trace except $P_{[l]} \otimes P_{[0,1]}^{\otimes m}$ are symmetric under conjugation, so $P_{[l]} \otimes P_{[0,1]}^{\otimes m}$ can be replaced by its symmetrised version $\overline{P_{[l]} \otimes P_{[0,1]}^{\otimes m}}$. Now we can insert eq. (90). The only term on the right-hand side that does not vanish when projected by $\mathcal{P}^{\otimes n}$ to a representation of $S U(2)$ is $J=[l, m, 0, \ldots]$ with multiplicity 1 . The dimensions of the representations $[l]$ and $[0,1]$ (of the symmetric group) are 1 , while the dimension of $[l, m]$ appearing in the denominator of (90) just cancels that on the right-hand side of (38), so we obtain the left-hand side. 
We compute the product of multiple (anti-)holomorphic derivatives of $\omega_{L}$ and $\omega_{L}^{\prime}$ projected to the representation $\left([l, m]^{*},[l, m]\right)$ of the stability group,

$$
X_{l, m}^{(L)} \equiv\left(\partial_{A_{1}} \cdots \partial_{A_{l+2 m}} \omega^{L}\right) K_{[l, m]}^{A_{1} \ldots A_{l+2 m}, B_{1} \ldots B_{l+2 m}}\left(\partial_{B_{1}} \cdots \partial_{B_{l+2 m}} \omega^{L L}\right) .
$$

By eq. (91) and since $K_{[l, m]}$ contains the projector $K$, the derivatives in this equation are really covariant derivatives, holomorphic ones acting on $\omega^{\prime}$ and anti-holomorphic ones on $\omega$. Equation (38) implies that $K_{[l, m]}$ can be replaced by $d_{[l, m]} K_{[l]} \otimes K_{[0,1]}^{\otimes m}$,

$$
X_{l, m}^{(L)}=d_{[l, m]}\left(\partial^{l+2 m} \omega^{L}\right)\left(K_{[l]} \otimes K_{[0,1]}^{\otimes m}\right)\left(\partial^{l+2 m} \omega^{\prime L}\right)
$$

where we have introduced an index-free notation. Using the second equality of eq. (41), we find

$$
K_{[0,1]}^{A B, C D} \partial_{C} \partial_{D} \omega^{L}=\frac{L(L+1)}{2} \omega^{L-1} K_{[0,1]}^{A B, C D} \partial_{C} \partial_{D} \omega
$$

which iterates to

$$
\left(K_{[0,1]} \partial \partial\right)^{m} \omega^{L}=\frac{L !(L+1) !}{(L-m) !(L+1-m) !} \omega^{L-m}\left(\frac{1}{2} K_{[0,1]} \partial \partial \omega\right)^{m}
$$

because the triple derivative of $\omega$ vanishes. The first equality of eq. (41) implies that $K_{[l]} \partial^{l} \omega^{n}$ contains only single derivatives of $\omega$, whence

$$
\begin{aligned}
\left(K_{[l]} \otimes K_{[0,1]}^{\otimes m}\right) \partial^{l+2 m} \omega^{L}=\left(K_{[l]} \otimes K_{[0,1]}^{\otimes m}\right)\left[\partial^{l}\left(K_{[0,1]} \partial \partial\right)^{m} \omega^{L}\right] \\
=\frac{L !(L+1) !}{(L-l-m) !(L+1-m) !} \omega^{L-l-m}\left[K_{[l]}(\partial \omega)^{l}\right]\left[\frac{1}{2} K_{[0,1]}(\partial \partial \omega)\right]^{m}
\end{aligned}
$$

where, in the first step, we have used eq. (8). Since a similar equality holds for anti-holomorphic derivatives, and $K_{[l]}$ and $K_{[0,1]}$ are projectors, we find

$$
\begin{aligned}
X_{l, m}^{(L)}= & d_{[l, m]}\left(\frac{L !(L+1) !}{(L-l-m) !(L+1-m) !}\right)^{2}\left(\omega \omega^{\prime}\right)^{L-l-m} \\
& \times\left[(\partial \omega)^{l} K_{[l]}\left(\partial \omega^{\prime}\right)^{l}\right]\left[\frac{1}{4}(\partial \partial \omega) K_{[0,1]}\left(\partial \partial \omega^{\prime}\right)\right]^{m} .
\end{aligned}
$$

\section{References}

[1] A. Connes, Noncommutative Geometry, Academic Press (1994).

[2] J. Madore, An Introduction to Noncommutative Differential Geometry and its Physical Applications, Cambridge (1995); "Noncommutative geometry for pedestrians," gr-qc/9906059.

[3] N. Seiberg and E. Witten, "String theory and noncommutative geometry," JHEP 09 (1999) 032, hep-th/9908142. 
[4] H. Grosse, C. Klimčík and P. Prešnajder, "Towards finite quantum field theory in noncommutative geometry," Int. J. Theor. Phys. 35 (1996) 231, hep-th/9505175.

[5] U. Carow-Watamura and S. Watamura, "Chirality and Dirac operator on noncommutative sphere," Commun. Math. Phys. 183 (1997) 365, hep-th/9605003; "Noncommutative geometry and gauge theory on fuzzy sphere," Commun. Math. Phys. 212 (2000) 395, hep-th/9801195.

[6] A. P. Balachandran, T. R. Govindarajan and B. Ydri, "The fermion doubling problem and noncommutative geometry," Mod. Phys. Lett. A 15 (2000) 1279, hep-th/9911087; "The fermion doubling problem and noncommutative geometry. II," hep-th/0006216.

[7] H. Grosse, C. Klimčík and P. Prešnajder, "Topologically nontrivial field configurations in noncommutative geometry," Commun. Math. Phys. 178 (1996) 507, hep-th/9510083; P. Prešnajder, "The origin of chiral anomaly and the noncommutative geometry," J. Math. Phys. 41 (2000) 2789, hep-th/9912050.

[8] S. Baez, A. P. Balachandran, B. Idri and S. Vaidya, "Monopoles and solitons in fuzzy physics," Commun. Math. Phys. 208 (2000) 787, hep-th/9811169, A. P. Balachandran and S. Vaidya, "Instantons and chiral anomaly in fuzzy physics," Int. J. Mod. Phys. A 16 (2001) 17, hep-th/9910129;

[9] M. Kontsevich, "Deformation quantisation of Poisson manifolds," q-alg/9709040.

[10] F. A. Berezin, "General concept of quantization," Commun. Math. Phys. 40 (1975) 153; "Quantization in complex symmetric spaces," Math. USSR, Izv. 9 (1975) 341.

[11] A. M. Perelomov, Generalized Coherent States and their Applications, Springer (1986); "Coherent states for arbitrary Lie groups," Commun. Math. Phys. 26 (1972) 222.

[12] B. Kostant, in Group Representations in Mathematics and Physics, ed. C. T. Taam, Lecture Notes Phys, Vol. 6, Springer (1970), p. 237.

[13] A. A. Kirillov, Elements of the Theory of Representations, Springer (1976).

[14] J. Rawnsley, M. Cahen and S. Gutt, "Quantization of Kähler manifolds. I: Geometric interpretation of Berezin's quantization," J. Geom. Phys. 7 (1990) 45.

[15] M. Cahen, S. Gutt and J. Rawnsley, "Quantization of Kähler manifolds. II," Trans. Am. Math. Soc. 337 (1993) 73.

[16] A. V. Karabegov, "Pseudo-Kähler quantization on flag manifolds," Commun. Math. Phys. 200 (1999) 355. 
[17] H. Figueroa, J. M. Gracia-Bondía and J. Várilly, "Moyal quantization with compact symmetry groups and noncommutative harmonic analysis," J. Math. Phys. 31 (1990) 2664.

[18] A. P. Balachandran, B. Dolan, J. Lee, X. Martin and D. O'Connor, "Fuzzy complex projective spaces and their star-products," hep-th/0107099.

[19] J. Madore, "The fuzzy sphere," Class. Quant. Grav. 9 (1992) 69.

[20] P. Prešnajder, "The origin of chiral anomaly and the noncommutative geometry," J. Math. Phys. 41 (2000) 2789, hep-th/9912050.

[21] Peter Prešnajder, private communication.

[22] J. Schirmer, "A star product for complex Grassmann manifolds," q-alg/9709021

[23] M. Bordemann, M. Brischle, C. Emmrich and S. Waldmann, "Subalgebras with converging star products in deformation quantization: an algebraic construction for $\mathbf{C P}^{n}$," J. Math. Phys. 37 (1996) 6311, q-alg/9512019; "Phase space reduction for star-products: an explicit construction for $\mathbf{C P}^{n}$," Lett. Math. Phys. 36 (1996) 357, q-alg/9503004.

[24] D. E. Littlewood, The Theory of Group Characters, Oxford University Press (1950).

[25] G. de B. Robinson, Representation Theory of the Symmetric Group, Edinburgh University Press (1961).

[26] C. R. Hagen, A. J. Macfarlane, "Reduction of representations of $\mathrm{SU}_{m+n}$ with respect to the subgroup $\mathrm{SU}_{m} \otimes \mathrm{SU}_{n}$ "J. Math. Phys. 6 (1965) 1366.

[27] M. L. Whippman, "Branching rules for simple Lie groups" J. Math. Phys. 6 (1965) 1534.

[28] S. Sternberg, Group Theory and Physics, Cambridge (1994).

[29] A. O. Barut and R. Rączka, Theory of Group Representations and Applications, World Scientific (1986).

[30] B. P. Dolan, D. O'Connor and P. Presnajder, "Matrix $\varphi^{4}$ models on the fuzzy sphere and their continuum limits," hep-th/0109084.

[31] A. Y. Alekseev, A. Recknagel and V. Schomerus, "Non-commutative worldvolume geometries: Branes on SU(2) and fuzzy spheres," JHEP 09 (1999) 023, hep-th/9908040.

[32] G. Felder, J. Fröhlich, J. Fuchs and C. Schweigert, "The geometry of WZW branes," J. Geom. Phys. 34 (2000) 162, hep-th/9909030. 